\title{
Filiation et transfert d'objets scientifiques dans les écrits de recherche
}

Francis Grossmann, Agnès Tutin et Pedro Paulo Garcia Da Silva

\section{(2) OpenEdition}

Édition électronique

URL : http://journals.openedition.org/pratiques/1447

DOI : $10.4000 /$ pratiques. 1447

ISSN : 2425-2042

Éditeur

Centre de recherche sur les médiations (CREM)

Édition imprimée

Date de publication : 15 décembre 2009

Pagination : 187-202

Référence électronique

Francis Grossmann, Agnès Tutin et Pedro Paulo Garcia Da Silva, « Filiation et transfert d'objets scientifiques dans les écrits de recherche », Pratiques [En ligne], 143-144 | 2009, mis en ligne le 19 juin 2014, consulté le 19 avril 2019. URL : http://journals.openedition.org/pratiques/1447 ; DOl : 10.4000/ pratiques. 1447 


\title{
Filiation et transfert d'objets scientifiques dans les écrits de recherche
}

\author{
Francis Grossmann, Agnès Tutin, \\ Pedro Paulo Garcia Da Silva
}

\author{
Lidilem, E.A. 609, Grenoble III
}

\section{La notion de filiation scientifique et ses entours}

Parler de filiation scientifique conduit à établir l'existence d'une lignée, s'établissant sur la base d'affinités ou de courants plus ou moins institutionnalisés, et qui incluent un auteur dans une communauté. Certaines expressions consacrées sont utilisées fréquemment pour marquer cette inscription dans les pas de prédécesseurs : notre étude s'inscrit dans la lignée des travaux de X... ; à la suite de $X$, nous défendrons l'idée que, ou encore, de manière moins personnalisée, notre travail s'inscrit dans le cadre de la théorie de X...

Le marquage de la filiation s'effectue en référence à un paradigme épistémologique ou à un courant de pensée (l'intuitionnisme, le constructivisme, le behaviorisme, etc.), un domaine scientifique pré-construit, qui peut avoir des frontières plus ou moins larges (la linguistique de l'énonciation, la psychologie cognitive, les neurosciences, etc.). Elle peut aussi renvoyer à un auteur particulier, ou à un groupe - équipe de recherche, école de pensée -, avec lesquels le chercheur a des affinités, ou auquel il emprunte tout ou partie de son cadre théorique ou de la démarche méthodologique mise en œuvre. Pour 1'apprenti-chercheur, il s'agit là d'un aspect important, parce que l'explicitation de la filiation le conduit à mieux cerner sa propre identité de chercheur. Dans un travail antérieur (Rinck, Boch \& Grossmann, 2007), nous avons pu montrer que les doctorants se réfèrent moins que les auteurs confirmés à différents points de vue, mobilisent moins de noms d'auteur, et se réfèrent moins à des courants particuliers, étiquetés sous des formes telles que le structuralisme, les fonctionnalistes, etc. Ce déficit s'explique principalement par la difficulté qu'éprouvent les nouveaux entrants dans le champ académique à trouver les moyens d'une véritable affiliation, permettant leur propre positionnement. Au-delà ou en deçà de la filiation, qui concerne directement l'identité du chercheur, se pose le problème plus général de l'intégration dans l'écrit de recherche d'« objets scientifiques » (désormais OS) aussi divers qu'une démarche, une méthodologie ou un cadre théorique issus des travaux des prédécesseurs ou des pairs. Il nous a paru intéressant de traiter les deux aspects conjointement, dans la mesure où il n'y a pas rupture mais bien continuité entre la filiation comme reven- 
dication d'un héritage intellectuel et les transferts de connaissance qui s'effectuent d'un chercheur à l'autre, à partir de différents types d'OS.

Une approche lexicale et phraséologique ne fournit certes pas toutes les clés d'un tel processus qui exige à la fois le renforcement des connaissances scientifiques dans le champ considéré, le dépassement de l'insécurité propre au néophyte, et l'intégration dans une communauté de discours, telle que la définit Swales (1990), mais également suivant la perspective un peu différente de la tradition française d'analyse de discours ${ }^{(1)}$. Elle permet cependant de mieux cerner la manière dont les auteurs confirmés procèdent, et surtout elle donne des outils permettant d'analyser les fonctions de la filiation dans l'écrit scientifique. Nous pensons par conséquent qu'elle peut offrir aux jeunes chercheurs des éléments utiles pour mieux comprendre l'importance de la construction d'une identité de chercheur, surtout si on la combine à une approche énonciative, précisant les modalités de la prise en charge et du positionnement dans l'écrit scientifique.

Nous limiterons ici la notion de filiation scientifique - ainsi que celle, connexe, de transfert de connaissances - pour éviter de les assimiler à l'ensemble des procédés relevant de la polyphonie ${ }^{(2)}$. En effet, bien que cette notion reste pour nous essentielle comme cadre général d'analyse, elle déborde largement notre propos, puisqu'elle intègre l'ensemble du processus citationnel ${ }^{(3)}$, alors que nous nous en tenons aux reprises de notions liés à l'épistémologie scientifique ${ }^{(4)}$.

Dans notre approche, la notion de filiation est limitée aux cas bien précis dans lesquels l'auteur du texte scientifique, en se référant à un auteur, ou à un courant théorique, ou encore à une école de pensée fortement attachée à un auteur ou groupe exprime explicitement à leur égard une forme de dette intellectuelle. Il arrive en effet très souvent que la filiation se marque de manière implicite, en particulier à travers le jeu des références, qui lorsqu'elles convergent vers un auteur ou une école marquent par là même, indirectement, l'inscription dans une certaine mouvance intellectuelle. L'exemple suivant en fournit un exemple :

(1) On partira ici de deux formulations de cette hypothèse, a priori fort difficilement réductibles, celle de Gustave Guillaume et celle d'Edmund Husserl, pour montrer comment elles aboutissent à des problématisations secondaires étrangement similaires. Gustave Guillaume est en effet un des rares linguistes à avoir sérieusement considéré l'hypothèse que l'endophasie soit radicalement non-communicationnelle. [KIAP - linguistique.]

(1) Pour une synthèse voir Beacco, 1995, Chareaudeau et Maingueneau, 2002 (entrée «Communauté discursive ») et Oger, 2005.

(2) Exploitée pour le discours scientifique, entre autres, par Fløttum et al. (2006) pour traiter les citations dans les textes scientifiques, dans lesquels la fonction de la polémique et du positionnement sont centrales. Se situant dans le cadre de la Scapoline (Nølke, Fløttum, \& Norén., 2004), Fløttum (2005, voir aussi 1992), dégage la présence d'un locuteur privilégié organisant une hiérarchie des voix multiples, ce qui différencie sa conception de celle de Bakthine (celui de la Poétique de Dostë̈vski plus que celui de Marxisme et Philosophie du Langage, et l'inscrit dans la continuité de Ducrot, 1984).

(3) «Citation » s'entend ici dans le sens général d'appui sur le discours d'autrui, incluant la reformulation.

(4) Il en va de même pour le concept d'intertextualité. Certes, les marques de la filiation, en plaçant le texte sous le patronage de maîtres, de chefs d'école ou d'auteurs connus, l'inscrivent bien comme l'un des procédés constitutifs du processus historique de l'intertextualité. Il ne s'agit pas ici d'étudier de manière générale ce tissage qui s'effectue avec les textes du dehors, mais de l'envisager exclusivement à partir d'un certain type de marques, et pour un certain type d'objets, nos « objets scientifiques ». 
En présentant les formulations de deux auteurs comme intéressantes et en indiquant l'originalité d'un des deux auteurs, Gustave Guillaume, l'auteur de l'article signale implicitement une affinité intellectuelle avec ce dernier ; 1'expression verbale on partira ici de représente déjà cependant une forme explicite de marquage, signalant le fait que les auteurs cités constituent un point de départ pour la réflexion du chercheur. Que l'on ait affaire à des formes implicites ou explicites de la filiation, il y a donc mise en relief de l'auteur cité non comme un auteur en plus parmi tous les autres cités, mais comme un auteur dont la contribution théorique ou méthodologique est indispensable pour l'objet théorique qui se construit dans l'article, et ce que ce soit pour un besoin ponctuel ou pour l'article dans son ensemble.

Nous nous intéresserons donc quant à nous préférentiellement au geste d'inscription explicite, à la fois pour des raisons pratiques (limité généralement au cadre de l'énoncé, il est plus facile à repérer), et pour des raisons théoriques (en tant qu'acte assumé il nous semble plus significatif, et il engage un positionnement plus net). Il reste qu'il serait également très intéressant d'étudier la forme implicite, qui est sans doute maniée plus habilement par les experts.

\section{Méthode d'analyse et corpus}

La manifestation de la forme explicite de la filiation scientifique et des transferts de connaissance dans les articles scientifiques présuppose les quatre catégories suivantes:

- La place énonciative correspondant à la figure de l'auteur, producteur de contenu scientifique, dont on peut observer les traces énonciatives à travers les indices personnels (nous, je, on ...) et/ou des substituts lexicaux de l'auteur (notre/mon/ce travail, article, approche...);

- Un processus d'appropriation/reprise qui se traduit lexicalement à travers des expressions verbales telles que se situer dans (la lignée de), utiliser, mobiliser, reprendre, recourir à, se référer à, prolonger; ou se marque par des locutions prépositives : à la suite de $X$.

L'«objet » scientifique repris : modèle, idée, définition, concept, terme, théorie, méthodologie...

- L'auteur convoqué ou ses substituts (école, approche, etc.), à qui est parfois prêté, mais pas obligatoirement ${ }^{(5)}$ un certain contenu énonciatif.

Notre corpus, issu du corpus KIAP, se compose d'un sous-corpus de 50 articles en linguistique (286.000 mots) et d'un sous-corpus de 50 articles en économie ${ }^{(6)}$ (374.500 mots). Le corpus de linguistique est constitué d'articles de linguistique générale et de sémantique, tirés des revues suivantes : Langue Française (13 articles de 2001-2002), Marges Linguistiques (2 articles de 2001), Revue de Sémantique et Pragmatique (17 articles de 1999-2001), Travaux de Linguistique (18 articles de 2001-2002). Le corpus d'économie se décompose de la façon suivante : Annales d'Économie et de Statistique (34 articles de 1998 à 2001), Économie Ap-

(5) Le nom d'auteur ou d'école joue parfois le rôle d'emblème de position (Grossmann, 2002, 2003), activant les connaissances partagées dans la communauté discursive : dans ce cas, il n'y a pas obligatoirement rappel de la théorie ou du contenu conceptuel prêté à l'auteur.

(6) http ://www.uib.no/kiap/KIAPCorpus.htm. Merci à Kjersti Fløttum et à son équipe d'avoir accepté de mettre à notre disposition ce corpus, celui que nous constituons dans le cadre du projet Scientext étant encore en cours d'élaboration. 
pliquée ( 7 articles de 2000 et de 2001), Revue Économique (9 articles de 2000 et 2001). Le corpus d'économie est formé de revues également plutôt généralistes, et bien connues dans le domaine francophone, les revues les plus prestigieuses en économie étant toutes anglophones.

Nous observons que dans les exemples 2 à 6 ci-dessous les quatre catégories que nous avons proposées apparaissent de la manière suivante : l'auteur producteur de contenu scientifique est représenté par nous (2), (3) et (6) ou on (4) et (5); les mécanismes d'appropriation apparaissent à travers proposons [ici] de recourir à(2), reprenons (3), [propose ici] d'utiliser (4), utilise (5) et retenons (6) ; les objets scientifiques qui font l'objet d'une appropriation sont théorie [des clubs] (2), définitions (3), procédure [convergente] (4), méthode (5) et démarche pragmatique (6); le système auteur-date est utilisé partout sauf en (3) et en (6), les dates figurant en amont ou en aval.

(2) Nous proposons ici de recourir à la théorie des clubs de Buchanan [1965] : d'une part, car le club est un moyen de traiter de la consommation socialisée en restant dans le champ analytique de l'économie; d'autre part, parce que des applications empiriques sont possibles à travers la consommation partagée de biens durables. [Corpus KIAP Economie]

(3) Nous reprenons ici les définitions proposées par K. Lambrecht pour lequel le topique et le focus sont des relations pragmatiques : the pragmatic relation between a topic and a proposition is assumed to be predictable or recoverable, the relation between the focus element and the proposition is assumed to be unpredictable or non-recoverable for the addressee at the time of the utterance. (Lambrecht, 1994 : 217) [Corpus KIAP Linguistique]

(4) On se propose ici d'utiliser une procédure convergente décrite par CHAMBERLAIN [1984] afin de comparer les résultats avec ceux obtenus en coupe instantanée. [Corpus KIAP Economie]

(5) Afin de disposer d'estimateurs convergents, on utilise une méthode proposée par CHAMBERLAIN [1984] qui consiste à se ramener à un modèle dans lequel l'espérance des effets individuels est linéaire par rapport aux variables explicatives. [Corpus KIAP Economie - même article de l'extrait antérieur]

(6) Ainsi, nous retenons la démarche pragmatique proposée par Fleurbaey et Michel en termes de courbes de niveau moyen en fonction de l'âge. [Corpus KIAP Economie]

Remarquons qu'il arrive que les auteurs de l'article explicitent la filiation d'un autre auteur mais pas leur propre filiation. Toutefois, notre propos ici se restreint volontairement à donner quelques pistes sur ce qu' on pourrait appeler la « filiation primaire ». L'exemple (7) montre une manifestation de ce type de filiation secondaire non considéré dans notre étude :

(7) Il convient de rappeler ici qu'à la suite de Milner (1978), beaucoup de chercheurs établissent une distinction fondamentale entre en quantitatif [5-6] et les autres emplois de en, dont en adnominal [7-8]. [KIAP - linguistique]

Dans cet exemple, l'auteur renvoie à la filiation des chercheurs qui se situent à la suite de Milner par rapport aux usages de en. L'auteur de l'article, toutefois, ne se situe pas dans cet ensemble. Nous avons exclu des nos résultats toutes les occurrences de ce type, qui marquent une filiation secondaire. 


\section{Les marques de la filiation et du transfert dans le corpus}

Nous avons rencontré 60 occurrences de la filiation et du transfert dans le souscorpus d'articles en économie et 30 occurrences dans le sous-corpus de linguistique. Ce résultat indique une différence de portée du phénomène dans ces deux disciplines, nettement plus représenté dans le sous-corpus d'économie (nous revenons en 4.4 sur ces différences disciplinaires).

C'est pour l'auteur producteur de contenu scientifique que la variété des formes est la plus limitée. Dans cette catégorie, on observe la forte prépondérance des on et nous, et bien plus rarement je. Nous ne reviendrons pas ici sur les valeurs énonciatives de on vs nous dans l'écrit académique et de recherche, qui ont fait l'objet de nombreux développements dans la littérature (voir par exemple Kinn, 2005, Gjesdal, 2008, Fløttum, Jonassen \& Norén, 2007).

Notons que le marquage de la filiation peut se passer des formes on ou nous (voire je) en se référant plus directement à l'article scientifique donné à lire (par exemple : cet article s'inscrit dans la théorie de X). D'après nos observations en corpus, ce cas de figure n'est cependant pas si fréquent, et apparaît moins que les formes correspondantes avec on et nous ou avec des groupes nominaux accompagnés de possessifs qui renvoient à l'auteur (ou aux auteurs) comme dans notre approche. L'article lui-même est souvent instancié grâce à des déictiques comme $i c i$. Ainsi, en (2), (3) et (4) l'auteur est représenté par on ou par nous mais une localisation s'effectue grâce au terme ici. Constatons aussi que notre choix de nous en tenir à la forme explicite de la filiation a pour conséquence la prépondérance de reprises ponctuelles, concernant en particulier les exemples et le corpus utilisé comme on verra plus loin. Certaines formes verbales (nous proposons de...) permettent à l'auteur de marquer ses choix et de les expliciter dans l'interaction avec le lecteur.

Les OS ont pour correspondants des «noms scientifiques », qui ont une fonction importante dans tous les domaines scientifiques. Quelques-uns, comme théorie, restent assez éloignés de la langue non spécialisée. D’autres, comme définition ou démarche, apparaissent plus facilement dans des usages langagiers non scientifiques. Ces noms sont majoritairement des construits abstraits de l'activité scientifique, par opposition aux observables (données, résultats) ${ }^{(7)}$. Les termes théorie [des clubs] (2), définitions (3), procédure [convergente] (4), méthode (5) et démarche [pragmatique] (6), relèvent de la «Langue Scientifique Générale » (Pecman, 2007), ou du « Lexique Scientifique Transdisciplinaire (LST)» (Drouin 2007 ; Tutin 2007a : Tutin 2007b), ce qui explique qu'ils aient des acceptions différentes suivant les disciplines et les contextes.

Les problèmes lexicaux évoqués ci-dessus sont récurrents pour le lexique relatif aux OS. On sait que les sciences ou disciplines constituent leurs objets d'étude dans leurs textes à travers des objets spécifiques. Ainsi, théorème concerne plus spécifiquement les mathématiques, et reste en dehors du lexique scientifique général (ou du moins, n'a pas un usage aussi général et répandu que d'autres termes). Théorie bien que moins polysémique dans les textes scientifiques qu'hypothèse et postulat (Cavalla et Grossmann, 2005 : 55), comporte au moins deux grandes interprétations, celle de «construction synthétique » ou celle de «système servant de base pour l'observation empirique ». On peut donc comprendre que, dans l'ar-

(7) Sur le plan linguistique, ces noms abstraits ont toujours un agent humain et sont non extensifs au sens de Flaux et van de Velde (2000). Ils se combinent naturellement avec des verbes comme élaborer, construire. 
gumentation, il puisse être utilisé soit dans le sens de système pré-construit, soit dans son acception synthétique, ce qui n'empêche pas qu'il reste central dans tous les domaines scientifiques.

\section{Le marquage de la filiation et ses fonctions pragmatiques}

Notre hypothèse de travail peut s'énoncer de la manière suivante : le marquage de la filiation représente une étape obligée vers un positionnement de l'auteur; il aboutit à un positionnement personnel, parfois démarcatif. Cette hypothèse peut sembler paradoxale, puisqu'il semble a priori que la reconnaissance d'une filiation s'apparente à une forme de refuge dans la sécurité des vérités établies, ou même à un simple recours à l'argument d'autorité.

\subsection{Filiation et argument d'autorité}

Présenter son travail dans une chaîne de filiation correspond-il à ce que la philosophie médiévale depuis Saint Augustin appelle l'argument d'autorité (pour une présentation, voir par exemple Raynaud, 1998) ? Il faut rappeler que l'argument d'autorité continue à avoir une certaine importance, dans les sciences contemporaines, si l'on considère le caractère cumulatif du travail scientifique : puisqu'il faut s'appuyer sur les travaux antérieurs, les critères de reconnaissance académique et l'autorité des publications reconnues continuent, généralement à bon droit, de justifier l'appui sur les pairs et les prédécesseurs. Cependant la place principale est donnée, en principe, aux appuis empiriques, et ce dans tous les domaines de la connaissance, ce qui invalide le procédé par lequel certains étudiants et néophytes dans la recherche l'utilisent comme une « roue des secours » permettant de pallier un manquement empirique.

Dans les sciences humaines et sociales, les marques de la filiation, même les plus ponctuelles, ne présentent un intérêt que lorsqu'elles ont une implication forte pour la construction même du raisonnement, ainsi qu'en témoigne l'exemple (8) :

(8) Le troisième regard qui sera présenté ici repose sur le modèle de la Morphologie Autonome, et, se prononçant pour la séparation des opérations syntaxiques et morphologiques, rend compte des différences observées entre la possessivation et les autres prises de position des génitifs. [...]

On fera donc fond sur deux axes : [...]

Le modèle théorique de la Morphologie Distribuée (ou Autonome), $c f$. Halle et Marantz (1993), Harris (1997), Zribi-Hertz (1998). On montrera, en le particularisant, que ce modèle s'applique sur un mode contrastif à son et à en : son, à la différence de $e n$, est un item fléchi, dont la formation à partir des traits flexionnels relève de la morphologie et non de la syntaxe.

En bref, pour reprendre les exposés de Harris (1997) et de Zribi-Hertz (1998), cette conception énonce que les items fléchis, dans notre cas son, ne sont pas insérés tout fléchis dans les configurations syntaxiques, mais résultent d'opérations morphologiques qui s'appliquent au produit des opérations syntaxiques.

[KIAP - linguistique. Les gras sont de notre fait, ainsi que dans les exemples suivants]

Dans le premier extrait, issu du résumé, on observe qu'ici renvoie à l'auteur producteur du contenu scientifique, que repose sur marque le mécanisme d'appropriation, que modèle représente l'objet scientifique qui fait l'objet de cette appropriation et que morphologie autonome représente le cadre ou l'approche dans le- 
quel l'auteur se situe. Dans le deuxième extrait, l'auteur de l'article reprend le modèle théorique auquel il se réfère dans le résumé et élucide l'intérêt de cet objet pour son article. Cet exemple démontre la visée potentiellement critique du marquage de la filiation.

On note un deuxième aspect, tout aussi important, qui consiste en une spécification de l'OS importé, ce qui se marque à travers des formulations comme « en le particularisant» et « dans notre cas » et conforte l'hypothèse selon laquelle le marquage de la filiation sélectionne les objets scientifiques qui sont centraux pour le développement de ses propres raisonnements scientifiques sans que cela présuppose toujours une adhésion au cadre théorique d'ensemble. L'objet scientifique est alors inséré dans l'ensemble de l'article dans lequel il prend sens. Certains éléments ont justement pour fonction d'intégrer les OS dans l'ensemble du texte en explicitant leur importance pour l'article : de ce fait, ils apparaissent souvent dans l'introduction, comme c'est le cas en économie. On remarque également qu'ils apparaissent très souvent dans cette discipline, dans les résumés.

\subsection{Les différents types d'objets scientifiques «empruntés »}

Plusieurs types d'objets scientifiques sont « empruntés» dans le processus de filiation.

a) les objets scientifiques construits complexes (méthode, modèle, théorie, approche) renvoient à des objets abstraits construits par les chercheurs qui sont décomposables en plusieurs objets simples et possèdent une structure complexe (ils constituent un système).

b) les objets scientifiques construits simples ${ }^{(8)}$ (concept; notion, idée; définition) sont des contenus abstraits construits par un auteur (par exemple, en linguistique la notion de signe de Saussure, ou la définition de la référence chez Frege). Ces éléments ne constituent pas un système explicatif et/ou procédural comme les objets scientifiques construits complexes à eux seuls, mais peuvent bien entendu faire partie d'un objet scientifique construit complexe. Certains objets scientifiques simples peuvent être associés de façon plus ponctuelle aux écrits d'un auteur, comme dans l'exemple suivant :

(9) Nous reprenons les distinctions de Sueur (1979) : Ia : permission, Ib : capacité.

Enfin, certains de ces objets simples sont des termes, terminologies, dénominations, mais l'emprunt de ces éléments métalinguistiques s'accompagnant généralement du concept qui y est lié, comme dans (10), on peut les intégrer dans la classe des OS construits simples.

(10)Le second problème porte sur l'utilisation des résultats d'une exploration contextuelle et rejoint notre présupposé théorique. Il s'articule autour de l'appréhension du mot comme « unité de discours définie par son contexte » pour reprendre les termes de J. Dubois (1971), ou comme « unité de langue » dont les actualisations discursives « impliquent des variations de sens selon les contextes » (ibid.) [KIAP - linguistique]

c) Les objets scientifiques observés (les «observables ») : corpus, exemples, résultats, données. Ils peuvent avoir été construits, mais sont avant tout des objets

(8) Simples par leur unicité, mais évidemment souvent très complexes du point de vue de leur appréhension. 
examinés dans l'activité scientifique. Ils différent bien entendu largement d'un domaine à l'autre : examen d'éléments numériques en économie, exemples ou corpus en linguistique, comme dans l'exemple suivant :

(11)L'étude de tous les verbes n'étant pas envisageable dans le cadre de cet article, nous utiliserons le corpus constitué par P. Le Goffic et N. Combe McBride (1975) en nous limitant aux verbes intransitifs, car dans les constructions transitives il faut prendre en compte le complément, plus précisément le déterminant du complément, qui joue également un rôle dans l'acceptabilité [...]. [KIAP - linguistique]

\subsection{Remarques sur la polysémie de certains termes : modèle et travaux}

Un terme comme modèle est polysémique, puisqu'il apparaît aussi bien en tant qu'outil scientifique pour des mesures économétriques ou pour désigner constructions scientifiques plus complexes. On retrouve cette ambiguïté dans deux des définitions de modèle données par le Trésor de la Langue Française informatisé ${ }^{(9)}$ :

a) ÉCON. «Représentation schématisée et chiffrée de l'évolution économique d'un pays pendant une période donnée à partir de ses caractéristiques (démographie, circulation de la monnaie et des biens, profit, épargne, investissement, consommation, etc.) et des relations de cause à effet qui unissent ces variables »(Cida 1973).

$[\ldots]$

b) LING. Construction abstraite, rigoureusement axiomatisée, utilisable pour formuler une théorie linguistique. Modèle d'analyse.

La définition donnée par le TLFi pour modèle en économie - qui ne couvre certes pas tous les emplois du terme dans cette discipline - explique qu'un modèle entendu en ce sens, ou dans un sens proche, puisse facilement être transféré. L'emploi de modèle en linguistique est donc bien souvent différent de son utilisation en économie.

A l'inverse, le lexème travaux apparait de manière assez semblable en linguistique et en économie. En linguistique comme en économie, il s'agit d'un terme vague, qui permet de référer aussi bien à la littérature qu'à des aspects plus concrets (démonstrations, expérimentations, résultats obtenus, etc.) :

(12)La littérature économique consacrée à ce thème comprend CLARKE et REED[1994], CONRAD [1988], CROPPER [1976], GOTTINGER [1991], PETHIG [1991] et ROE et al. [1994]. Pour point de départ de notre discussion, nous retenons les travaux de GOTTINGER et PETHIG uniquement. [KIAP - économie]

(13)Précisons que nous considérerons, à la suite des travaux de M.-J. Reichler-Beguelin, que la relation d'anaphore ne consiste pas à dupliquer les antécédents, mais à garder la trace de leur interprétation antérieure qui évolue en fonction des nouvelles propositions ou propriétés qui leur sont attribuées. [KIAP - linguistique]

Dans ces deux exemples, travaux rend renvoie aux publications deux écrits de deux auteurs (en (12)) et de plusieurs écrits d'un seul auteur (en (13)). Dans l'exemple (13), le nom de l'auteur est mentionné sans références, ce qui donne un sens très général au lexème et l'éloigne de toute référence à des œuvres précises.

Cette catégorie de lexèmes ouverts à l'interprétation, est, par ailleurs, la plus représentative dans les deux disciplines de notre corpus, même si elle est davantage représentée en linguistique (19 cas, $63 \%$ ) qu'en économie (30 cas, $50 \%$ ).

(9) http ://www.cnrtl.fr/definition/mod\%C3\%A81e, consulté le 01/09/2009. 


\subsection{Le marquage de la filiation dans les deux disciplines}

Si l'on compare à présent les deux disciplines, on observe, outre le fait que la filiation explicite est bien plus marquée en économie (60 occurrences de filiation explicite dans cette discipline, contre 30 seulement en linguistique), des différences marquantes dans les types d'objets empruntés.

Les OS construits complexes (modèle (11 occ.), méthode (4 occ.), approche (4 occ.), théorie ( 2 occ.) sont en effet bien plus fréquents en économie qu'en linguistique, où dans cette classe d'OS, on ne relève que modèle (2 occ.). Les emprunts théoriques manifestes en linguistique paraissent plus ponctuels, et moins structurés. On relève des OS construits simples comme notion (2 occ.), conception ( 2 occ.), distinctions ( 2 occ.) ou de termes plus généraux (travaux : 3 occ). On relève des emprunts terminologiques dans cette discipline (terme, terminologie, nom : 1 occ. de chaque élément), ce qui n'apparaît pas en économie.

Le repérage de ces éléments permet d'éclairer sur la façon dont la transmission des idées et des savoirs est explicitement exprimée par les chercheurs dans ces deux sciences humaines et sociales. Les économistes utilisent davantage de termes ouvertement métascientifiques (comme modèle, méthode, approche) qui donnent clairement un affichage scientifique à leur production. Le fait que les OS construits complexes sont transmissibles et empruntables d'un chercheur à l'autre montre que la discipline construit des objets structurés " prêts à l'emploi », s'ils apparaissent valides, et qu'il y a un fonctionnement cumulatif des appareillages théoriques ou méthodologiques développés. En linguistique, dans notre corpus, l'approche de la transmission scientifique apparaît moins structurée. Ce ne sont pas tant des OS complexes et structurés qui sont empruntés, mais plutôt des concepts plus ponctuels (concepts, notions, distinction, définition). Les linguistes mentionnent très peu l'emprunt à des modèles structurés déjà existants. Plusieurs explications, non exclusives, peuvent être données à cela. Tout d'abord, on peut penser que dans cette discipline des sciences humaines, il est probablement valorisé, surtout dans des revues prestigieuses comme celles qui apparaissent dans le corpus, d'affirmer son propre point de vue, plutôt que de situer dans le sillage d'autrui, ce que l'emprunt à un modèle théorique " prêt à l'emploi » laisserait entendre. Deuxièmement, il est probable que nombre de travaux en linguistique générale ne s'inscrivent pas dans des modèles ou théories linguistiques structurées formellement. Ce caractère formel peut se rencontrer dans certains travaux de grammaire générative ou de sémantique formelle, mais ils sont très peu représentés dans le corpus étudié.

Si les économistes tendent à inscrire plus nettement leur filiation dans les écrits scientifiques, ils se positionnement aussi davantage par rapport aux pairs. Une étude effectuée sur le même corpus par Chavez (2008) montre ainsi qu'ils tendent à employer davantage de marques de démarcation par rapport aux pairs que les linguistes, en particulier dans la discussion sur les modèles et les approches.

(14) Contrairement à Andreoni et Levinson [2001], notre objectif n'est pas ici de déterminer les conditions nécessaires et suffisantes sur les préférences ou sur les technologies de dépollution qui assurent l'existence d'une courbe environnementale de Kuznets. En dehors du fait que les formes fonctionnelles retenues pour l'utilité et la technologie sont particulières, le modèle analytique que nous proposons considère, comme dans Andreoni et Levinson [2001], des émissions liées au niveau de consommation. [KIAP - économie]

En outre, ils tendent aussi à davantage utiliser de lexique évaluatif que les lin- 
guistes (Tutin à paraître), en particulier pour qualifier leurs approches et celles des pairs (approche nouvelle, modèle traditionnel, nouveau modèle) et emploient plus systématiquement des pointeurs métatextuels indiquant les points saillants de leur contributions. Exemple : trois principaux résultats ont été dégagés). Les économistes tendent donc à davantage mentionner les objets scientifiques, qu'il s'agisse d'indiquer l'origine, la différence de point de vue, ou de les évaluer (souvent d'ailleurs pour valoriser leurs modèles, dans une stratégie d'auto-promotion). En revanche, toujours sur le même corpus, Fløttum et al. (2006) montrent que les linguistes sont davantage «visibles » que les économistes, en faisant davantage mention d'eux-mêmes dans les textes, par le biais de pronoms de première personne. Les traces interpersonnelles, visant à se situer et à interagir auprès du lecteur, semblent donc davantage perceptibles en économie qu'en linguistique, où en revanche les traces du sujet-locuteur semblent plus manifestes.

Dans les exemples du type (15), les connecteurs finaux introduisent une justification de l'utilité instrumentale de l'objet scientifique auquel il est fait recours pour le développement exposé dans l'article. Ce type de justification apparaît plus souvent en économie qu'en linguistique. Il est possible que cela s'explique par une nécessité plus nette chez les économistes d'utiliser des objets scientifiques dont la fonction théorique ou pratique est préalablement définie, notamment dans le cas où il est nécessaire de quantifier des résultats, ou d'utiliser une forme spécifique de modélisation des données, comme cela apparaît en également en (15) :

(15)Pour estimer le modèle probit bivarié, on reprend une approche introduite notamment par MROZ [1999], consistant à redéfinir les termes d'erreur dans le modèle initial : [KIAP - économie]

Si l'on observe plus précisément le lexique verbal utilisé dans les mécanismes d'emprunt dans les deux disciplines ( $C f$. tableau 1), on recense les verbes suivants :

\begin{tabular}{|l|l|}
\hline Economie & Linguistique \\
\hline utiliser (9) & reprendre (7) \\
reprendre (5) & utiliser (3) \\
retenir (4) & emprunter (3) \\
suivre (4) & suivre (3) \\
fonder sur (3) & renvoyer (2) \\
s'inspirer (3) & rejoindre (2 \\
s'inscrire (3) & \\
recourir (2) & \\
adopter (2) & \\
s'appuyer (2) & \\
\hline
\end{tabular}

Tableau 1 : Principales expressions (et nombre d'occurrences) utilisées pour les mécanismes d'emprunt en économie et en linguistique (fréquence $>1$ )

Le lexique verbal utilisé pour marquer l'emprunt est plus diversifié en économie, et le verbe utiliser est plus fréquent, ce qui rejoint notre observation précédente sur les OS, davantage structurés et «prêts à 1'emploi » en économie. Les marques de la filiation par héritage (voir 4.5), sont également mieux représentées dans le sous-corpus d'économie, comme le montre la présence du verbe le plus prototypique de cette catégorie, s'inscrire (dans...), absent de la partie linguistique comme marque de filiation (même si nous en avons trouvé des emplois, hors corpus). Le verbe reprendre est utilisé plus fréquemment en linguistique qu'en 
économie pour les reprises métalinguistiques ou terminologiques ( 1 seul emploi de ce type en économie, contre 5 en linguistique). On peut faire la même remarque pour le verbe emprunter ( 3 emplois en linguistique, pour signaler des emprunts de terminologie, 1 seul en économie).

\subsection{Deux aspects du fonctionnement pragmatique : de l'« utilisation " à l'« héritage "}

Nous distinguerons, de manière sans doute un peu grossière, deux aspects complémentaires, qui n'épuisent pas bien entendu les fonctions pragmatiques de la filiation, mais se traduisent assez nettement à travers les marques lexicales. Le premier met en scène la dimension fonctionnelle des transferts de connaissance, tout en garantissant la traçabilité des références. Le deuxième manifeste davantage l'inscription dans une communauté discursive scientifique, en signalant l'héritage que veut faire fructifier le chercheur. Dans les deux cas, les formes d'appropriation peuvent varier sensiblement.

Observons les trois exemples illustrant ce que nous avons appelé « utilisation » :

(16)4. Le modèle de négociation que nous utilisons est dû à Cahuc et Zylberberg [1999]. [KIAP - économie]

(17) On empruntera à C. Kerbrat-Orecchioni (1980) le nom de cotexte en l'infléchissant légèrement, dans la mesure où on l'utilisera pour désigner de manière restrictive ce qui relève de l'unité phrastique, de la distribution et on réservera le nom de contexte à tous les éléments cognitifs, situationnels ou intertextuels, susceptibles d'intervenir dans le processus de construction ou d'identification du sens. [KIAP - linguistique]

(18)Conformément aux recommandations de Searle (1985), nous utilisons la majuscule chaque fois que le mot «Intentionnel », ou l'un de ses dérivés, ne s'emploie pas dans son usage ordinaire : tout ce qui est intentionnel est Intentionnel, mais non réciproquement. [KIAP - linguistique]

Dans 1'exemple (16), l'objet scientifique (modèle) accompagne le verbe utiliser, signalant un emprunt, sans plus de précision. En (17), l'OS terminologique est employé avec une spécification (en l'infléchissant légèrement) qui ouvre un espace de positionnement, qui reste cependant très circonscrit à l'usage terminologique. En (18), nous avons une situation un peu plus complexe, dans laquelle l'OS terminologique «Intentionnel » subit une prescription recommandée (par l'auteur cité, Searle). Nous observons que le mécanisme d'appropriation a ici des fonctions pragmatiques relativement différentes, bien qu'il s'agisse dans tous les cas de marquer avec précision, conformément à l'ethos scientifique, la démarche suivie, au plan théorique (emprunt d'un modèle restitué à ses auteurs), ou au plan terminologique (précision fournie sur l'usage d'un terme utilisé). Il est intéressant de noter qu'à côté de ces OS terminologiques - souvent introduits par des classifieurs comme terme ou terminologie - peuvent figurer des citations entre guillemets qui expliquent la terminologie empruntée et son intérêt pour 1'article, tout en l'intégrant à l'ensemble textuel. Nous avons trouvé dans le sous-corpus de linguistique six reprises d'OS signalés comme emprunts terminologiques et trois citations. En économie, nous avons trouvé quatre reprises terminologiques dont une comprenait aussi une citation plus longue :

(19)Les modèles de croissance durable retiennent comme norme de soutenabilité le maintien d'un niveau de consommation par tête au minimum constant 2 : nous nous 
situons dans ce que Méral [1997] appelle « une logique technologique», où « seules les conditions de production sont mises en avant». [KIAP - économie]

L'emprunt terminologique est signalé par le verbe appelle, le sens du terme étant explicité par la citation qui suit. Nous avons trouvé d'autres exemples, comme en (20) dans lequel la citation conduit à définir un terme :

(20)Le second problème porte sur l'utilisation des résultats d'une exploration contextuelle et rejoint notre présupposé théorique. Il s'articule autour de l'appréhension du mot comme « unité de discours définie par son contexte " pour reprendre les termes de J. Dubois (1971), ou comme « unité de langue » dont les actualisations discursives « impliquent des variations de sens selon les contextes» (ibid.) [KIAP - linguistique]

On peut évidemment penser qu'on se trouve ici à la périphérie du marquage de la filiation en tant que telle, puisqu'il s'agit ici d'emprunts très limités. Cependant, ce type de reprises terminologiques joue souvent le rôle d'indice pour l'inscription dans une filiation : on n'emprunte pas impunément les mots - et les définitions - d'autrui.

Les exemples relevant plutôt du second type, l'héritage, peu fréquents dans notre corpus, sont identifiables à travers le lexique nominal (lignée, héritage, tradition, continuité) ou à travers des locutions prépositives (à la suite de...), le lexique verbal étant plutôt utilisé pour marquer le positionnement, notamment à travers des structures locatives. Nous nous limiterons ici à illustrer deux tendances principales.

La manière, apparemment la plus simple consiste pour un auteur à se situer clairement dans le cadre théorique d'un auteur/courant, ce qui n'empêche nullement, par ailleurs l'expression d'un positionnement personnel, ni même la démarcation, ainsi qu'en témoigne 1'exemple suivant (hors corpus) :

(21) Quant à nous, nous ne retiendrons pas la conception carnapienne de l'intension, laquelle a donné, comme on sait, naissance à l'actuelle « logique intensionnelle », développée entre autres par R. Montague (1974). Nous nous situons, d'une part, dans la tradition ouverte par la Logique de Port Royal (avec la notion que Arnault et Nicole appelaient « compréhension » opposée à l'« étendue » ou extension) et par Leibniz et, d'autre part, nous acceptons pleinement l'héritage du «concept » chez G. Frege (1883). Nous partons donc de la définition suivante [...] (JeanPierre Desclés, Réseaux sémantiques : la nature logique et linguistique des relateurs, Langages, 22, 1987).

On constate qu'une démarcation initiale précède ici l'inscription dans un courant, l'auteur définissant ses propres positions à partir non pas d'un seul courant (la tradition ouverte par la logique de Port Royal) mais en référence également à d'autres auteurs (Leibniz et Frege). On dépasse donc ici le classique positionnement d'école, et un tel procédé semble typique de l'expert, dans la mesure où il exige recul historique et maîtrise du champ conceptuel, condition préalable à l'expression d'un point de vue propre. L'accord comme la démarcation s'expriment nettement à travers des marques (nous acceptons pleinement, nous ne retiendrons pas), ce qui témoigne d'un positionnement fort. La filiation se marque à travers les lexèmes tradition et lignée.

Un autre procédé possible dans cette première démarche d'inscription dans un cadre est la démarcation partielle à partir d'un cadre accepté, comme le montre l'exemple suivant : 
(22) Notre modèle s'inscrit dans la lignée de celui proposé par Green et Zhou [1998]. À la différence de ces derniers, nous n'envisageons pas la détermination des prix mais nous introduisons les stocks de marchandises et la dimension temporelle du processus de production. [KIAP - économie]

La notion même de lignée autorise les variations et les modifications, maintenant un air de famille épistémologique commun aux différents modèles.

La deuxième démarche consiste pour un auteur à se situer non dans le cadre de l'auteur/courant auquel il fait référence, mais à présenter son propre cadre comme ayant une affinité théorique par rapport à ce cadre primaire. L'auteur ne se situe donc pas à proprement parler dans le cadre théorique d'un autre chercheur, mais se présente comme développant un cadre théoriquement proche, ou contigu.

Les exemples suivants (les marques du processus d'appropriation/transfert ont été mises en gras) manifestent cette tendance :

(23)Dans la mesure où le cadre développé par D. Corbin constitue, à notre connaissance, le principal modèle de morphologie dérivationnelle sur le français, c'est par rapport à lui que nous nous situerons dans la section 1. [...] Le débat (1) a déjà fait l'objet d'une longue argumentation par D. Corbin, en faveur de la conception associative, conception à laquelle nous souscrivons. [KIAP - linguistique]

(24)L'idée d'héritage fonctionnel présentée ici pourrait, par ailleurs, être mise en correspondance avec la notion de «propriété extrinsèque » de Cadiot et Nemo (1997), ou même avec celle de « homologation » de Lebas (1999). [KIAP - linguistique]

L'image qui est ici privilégiée, plutôt celle de la continuité ou de la révérence aux maîtres, est celle d'un auteur dialoguant avec ses pairs, et donc participant pleinement à la communauté discursive. En (23), le syntagme $c$ 'est par rapport à lui [= ce cadre] que nous nous situerons marque cependant bien une forme d'héritage, tandis qu'en (24) il se limite à ce qui est présenté comme une simple correspondance.

\section{Conclusion}

Notre étude nous a permis d'explorer la manière dont est lexicalisée ce que nous avons nommé la filiation scientifique, avec ses entours impliquant l'importation ou le transfert de notions au sein d'un article scientifique. A ce titre, l'étude de la filiation représente bien un poste privilégié d'observation de la polyphonie constitutive des textes scientifiques. Cependant, en partant de cette notion, on est conduit à mettre davantage l'accent sur les aspects épistémologiques, et sur la manière dont, linguistiquement, l'auteur d'un article gère l'espace conceptuel dans lequel il prétend situer son propos. De ce point de vue, l'étude de la filiation nous semble particulièrement intéressante pour sensibiliser l'apprenti-chercheur à la question de l'identité du chercheur, dont on sait qu'elle représente souvent un obstacle important dans l'écriture. Du point de vue lexical, il est intéressant d'observer la manière dont est exprimé le processus d'appropriation. Deux tendances se dessinent :

- l'auteur mobilise le lexique du renvoi ou de l'utilisation, ce qui met en évidence la fonction instrumentale du marquage de la filiation, mais aussi la cohérence de l'univers scientifique tel qu'il est construit dans l'espace notionnel de l'article ;

- l'auteur mobilise le lexique de l'héritage ou de la lignée, dans des structures locatives (ex. s'inscrire dans), ce qui le conduit à s'inclure pleinement lui-même dans la communauté de discours qui le concerne. 
Notre hypothèse de travail, selon laquelle le marquage de la filiation et du positionnement sont fortement liés nous paraît confirmée, à l'issue de l'analyse de corpus, en raison de l'utilisation concomitante des marques de positionnement et de celles qui marquent la filiation ou le transfert. Cependant, le positionnement, lorsqu'il s'agit d' ' utiliser » une notion empruntée à autrui reste souvent assez discret, et s'effectue davantage à travers des précisions terminologiques, ou des marques visant à spécifier en quoi la notion importée est intéressante pour le propos de l'auteur, et comment il va l'utiliser. La filiation marquant l'héritage, au moins dans notre corpus, apparaît moins fréquente. Il faudrait sans doute pouvoir différencier plus finement la nature des articles : un article de synthèse théorique nécessite sans doute davantage ce type de marquage.

En ce qui concerne les moyens lexicaux liés à l'expression de la filiation/du transfert, ils s'organisent syntagmatiquement suivant un cadre assez nettement isolable: l'Auteur-producteur de l'écrit scientifique vise à s'approprier un Objet Scientifique issu d'un Auteur (ou courant, théorie) appartenant à la communauté de discours. Un tel cadre, malgré ou à cause de sa simplicité, représente, pour les étudiants devant se familiariser à l'écriture de recherche, une entrée intéressante dans la problématique de la filiation.

Le deuxième thème ouvert à la réflexion didactique concerne ce que nous avons appelé les Objets Scientifiques (OS). Il s'agit de notions généralement héritées de l'histoire de chaque discipline, mais qui peuvent avoir également une valeur très générale comme le montre le cas de travaux. La dénomination de ces objets fait souvent débat. Les trois catégories repérées : OS construits complexe (faisant système), OS construits simples (entrant dans un système), OS observés (construits ou non construits), peuvent permettre de sensibiliser les jeunes chercheurs, de manière concrète, aux aspects épistémologiques sous-jacents à l'écriture scientifique et aux problèmes que pose l'importation des OS, en fonction de leur statut.

Enfin notre travail s'inscrit également dans le cadre de travaux de rhétorique contrastive (voir notamment Hyland, 2004) suivant les disciplines : nous avons trouvé deux fois plus d'occurrences en économie qu'en linguistique du marquage de la filiation/transfert, alors que le corpus était de même taille. L'étude de la filiation, des marques de démarcation et d'évaluation semble cependant montrer que dans cette discipline, il apparaît essentiel de se positionner explicitement par rapport aux pairs. On ne peut pas exclure non plus le fait que les économistes aient des besoins plus spécifiques, les obligeant à « importer » des modèles d'analyse ou des méthodes pour traiter le type de données qui est le leur. Une sensibilisation aux habitus disciplinaires, appuyée sur l'observation de corpus serait là encore utile aux jeunes chercheurs.

Au-delà de ces différences, il est intéressant de constater que la notion de filiation ne peut s'envisager qu'en prenant en compte la construction des réseaux notionnels dans chaque domaine de connaissance. 


\section{Bibliographie}

Bakhtine, M. (1970) [1931] : La Poétique de Dostoievski, Paris, Editions du Seuil. - (1977) [1929] : Marxisme et philosophie du langage, essai d'application de la méthode sociologique en linguistique. Paris : Les Éditions de Minuit.

BEACCO, J.-C. (1995) : « À propos de la structuration des communautés discursives : beaux-arts et appréciatif », dans J.-C. Beacco et S. Moirand, Les enjeux des discours spécialisés, carnets du Cediscor,

CAVAlla, C. \& Grossmann, F. (2005) : «Caractéristiques sémantiques de quelques "Noms scientifiques" dans l'article de recherche en français », Akademisk Prosa, 2, Romansk institutt, Universitetet i Bergen, 47-59.

Charaudeau, P. \& Maingueneau, D. (2002) : Dictionnaire d'analyse du discours, Paris, Seuil.

Chavez, I. (2008) : La démarcation dans les écrits scientifiques : Les collocations transdisciplinaires comme aide à l'écrit universitaire auprès des étudiants étrangers. Mémoire de Master 2, sous la direction de Cristelle Cavalla, Université Stendhal.

Drouin, P. (2007) : «Identification automatique du lexique scientifique transdisciplinaire », Revue française de linguistique appliquée, 12(2), 45-64.

Ducrot, O. (1984) : Le Dire et le dit, Paris, Editions de Minuit.

FLAUX, D. \& VAN DE VELDE (2000) : Les noms en français : esquisse de classement, Paris, Ophrys.

Fløтtum, K. (1992) : «Polyphonic structure », dans A. Lindeberg, N.E. Enkvist \& K. Wikberg (eds.), Nordic Research on Text and Discourse, Abo : Abo Academy Press, 161-172.

- (2005): "The self and the others - polyphonic visibility in research articles ", International Journal of Applied Linguistics, 15 (1), 29-44.

- (Ed) (2007) : Language and Discipline Perspectives on Academic Discourse. Cambridge : Cambridge Scholars Publishing, 14-3.

Fløttum, K., DAhL, T. \& KinN, T. (2006): Academic Voices - across languages and disciplines, Amsterdam, John Benjamins Publishing Company.

FløtTUM, K., JonAsSON, K., NoRÉN, C. (2007) : ON-pronom à facettes, Bruxelles, Duculot-De Boeck.

GJESDAL, A.M. (2008) : Étude sémantique du pronom ON dans une perspective textuelle et Contextuelle, Thèse de doctorat, Université de Bergen, consultée à l'adresse http ://hal.archives-ouvertes.fr/docs/00/33/53/06/PDF/VersionFinaleAllkopi.pdf, le 28/08/2009.

GROSSMANN, F. (2003) : «Du discours rapporté au discours autorisé, le maniement des noms d'auteur dans l'article en Sciences Humaines », Estudios de Lengua y Literatura francesas, 14, 9-26.

— (2002) : «Les modes de référence à autrui : l'exemple de la revue Langages », Faits de langue, 19, 255-262.

HYLAND, K. (2004) : Disciplinary discourses : social interactions in academic writing. University of Michigan Press, Ann Arbor.

KInN, T. (2005) : Plays of we-hood: What do we mean by we?, Akademisk Prosa, 3, Romansk institutt, Universitetet i Bergen, 129-142.

NølKe, H., FløtTum, K. \& NorÉN, C. (2004) : ScaPoLine. La théorie scandinave de la polyphonie linguistique, Paris, Kimé.

OGER, C. (2005): «L'analyse du discours institutionnel entre formations discursives et problématiques socio-anthropologiques », Langage \& société 2005/4, n 114 , 113-128.

PECMAN, M. (2007) : «Approche onomasiologique de la langue scientifique générale ", Revue Française de Linguistique appliquée, XII-2, 79-96. 
RAYNAUD, R. (1998) : «Les normes de la rationalité dans une controverse scientifique : le cas de l'optique médiévale », L'Année sociologique, 1998, 48 (2), 447-466.

RincK, F., Boch, F. \& GROSSMANN, F. (2007) : " Quelques lieux de variation du positionnement énonciatif dans l'article de recherche », dans P. Lambert, A. Millet, M. Rispail et C. Trimaille, eds., Variations au cour et aux marges de la sociolinguistique. Mélanges offerts à Jacqueline Billiez, Paris, L'Harmattan.

SWALES, J.M. (1990): Genre analysis, English in academic and research settings, Cambridge University press, 1990.

TUTin, A. (2007a) : «Autour du lexique et de la phraséologie des écrits scientifiques », Revue Française de Linguistique appliquée, 2007, XII-2.

- (2007b) : «Modélisation linguistique et annotation des collocations : une application au lexique transdiciplinaire des écrits scientifiques », dans S. Koeva, D. Maurel \& M. Silberztein (dir.) Formaliser les langues avec l'ordinateur : de Intex à NooJ, Presses Universitaires de Franche Comté, Besançon, 189-215.

— (à paraître) : «Evaluative adjectives in academic writing in the humanities and social sciences », Interpersonality in written academic discourse : perspectives across languages and cultures, 11-13 décembre 2008, Saragosse.

\section{PRATIQUES}

\section{PROCHAIN NUMÉRO}

\section{Recherches en didactique du français}

Le contexte politique et institutionnel propre à la France a accentué, ces dernières années, la difficulté de mener à bien une recherche spécifique en didactique du français : ostracisme ou clivage du domaine par les disciplines mères (linguistique, littérature) et les organes de valorisation de la recherche, double inscription institutionnelle de ses agents (formation des maître à l'IUfm, recherche à l'université), inertie, vague, contradiction ou régression des réformes touchant la discipline, déni par les décideurs des nécessités théoriques et pratiques d'une formation professionnelle de haut niveau. Paradoxalement, c'est ce climat de fragilisation et d'urgence qui incline à chercher un point de vue plus réflexif et distant au sujet des recherches en didactique du français.

L'objectif du numéro est double :

- Il s'agit de dresser, dans une étroite synchronie (couverture des années 2005-2010), un inventaire des recherches en didactique du français, par le biais des thèses et HDR soutenues et touchant aux problématiques du domaine; sont par exemple concernées l'histoire, la sociologie, la psychologie des pratiques professionnelles et scolaires ou celles des productions langagières et textuelles. L'étendue du champ des recherches possibles en didactique du français, combinée à la complexité et aux tensions des paradigmes convoqués, est, comme on le sait, l'un des obstacles à sa reconnaissance identifiée par les institutions de la recherche, et, finalement, à son efficience scolaire et sociale.

- Il s'agit également d'amorcer les synthèses et bilans critiques qui se dégagent des axes de recherche, des thèmes et des méthodologies qui ont actuellement cours en didactique du français, pour y repérer les tendances dominantes ou les manques les plus apparents. L'enjeu est, ce faisant, d'esquisser de nouveaux objets de connaissance et de relier des investigations savantes à des pratiques de classe que l'on voudrait moins discriminantes qu'elles ne le sont aujourd'hui. À cet égard, les bilans chercheront le cas échéant à préconiser des objets et des démarches de recherche, jugés nécessaires mais insuffisamment satisfaits par l'état des lieux établi. 